TRANSACTIONS OF THE

AMERICAN MATHEMATICAL SOCIETY

Volume 351, Number 10, Pages 4263-4280

S 0002-9947(99)02446-0

Article electronically published on July 1, 1999

\title{
A $K$ COUNTEREXAMPLE MACHINE
}

\author{
CHRISTOPHER HOFFMAN
}

\begin{abstract}
We present a general method for constructing families of measure preserving transformations which are $K$ and loosely Bernoulli with various ergodic theoretical properties. For example, we construct two $K$ transformations which are weakly isomorphic but not isomorphic, and a $K$ transformation with no roots. Ornstein's isomorphism theorem says families of Bernoulli shifts cannot have these properties. The construction uses a combination of properties from maps constructed by Ornstein and Shields, and Rudolph, and reduces the question of isomorphism of two transformations to the conjugacy of two related permutations.
\end{abstract}

\section{INTRODUCTION}

By showing that any two Bernoulli shifts of the same entropy are isomorphic, Ornstein proved that there are a large number of ergodic theoretical properties that a collection of Bernoulli shifts cannot have. Many families of transformations have been constructed to show the contrast between Bernoulli shifts and $K$ transformations. For example, Ornstein and Shields constructed an uncountable family of $K$ transformations of the same entropy, no two of which are isomorphic [10]. Ornstein's theorem also proves that a Bernoulli shift has roots of all orders. On the other hand, Clark exhibited a $K$ transformation which has no roots [1]. By a counterexample we mean a transformation, or a family of transformations, that has a property that Ornstein's theory implies that Bernoulli shifts cannot have. Many such counterexamples have been constructed with $K$ or zero entropy mixing transformations. The goal of this paper is to present a general method which will both create new $K$ counterexamples, and unify the theory of the previously known examples.

In his paper on minimal self joinings, Rudolph creates a family of transformations for which he can reduce the problem of isomorphism of two transformations in the family to the conjugacy of two corresponding permutations [13]. More than this, he enumerates all the isomorphisms between any two transformations in the family, and he presents a unified theory of zero entropy mixing counterexamples. In this paper we construct a family of transformations, indexed by permutations, which are $K$ and loosely Bernoulli transformations. We would like to control the isomorphisms between any two transformations in this family as well as Rudolph could. We will not succeed at this, but we will be able to associate a permutation with any isomorphism. This allows us to reduce the problem of isomorphism of two transformations to conjugacy of two permutations. In section 5 we will define

Received by the editors March 31, 1997.

1991 Mathematics Subject Classification. Primary 28D05; Secondary 28D20.

(C)1999 American Mathematical Society 
a class of factors of these transformations. We classify these factors up to isomorphism. Thus in sections 6 and 7 we are able to construct counterexamples with $K$ transformations for most of Rudolph's zero entropy counterexamples.

The $K$ transformations will be a combination of the Ornstein-Shields $K$ but not Bernoulli transformations [10], and the zero entropy transformations in [13] and [4]. The principle lemmas are Lemma 4.1 through 4.3. These lemmas help us to construct the permutation associated with an isomorphism and allow our proofs to proceed in the manner of the zero entropy counterexample machine proofs.

\section{Construction}

Our space $\Omega=(e, f, s, 0)^{\mathbf{Z}}$. The transformation $K$ is defined by $K(\omega)_{i}=\omega_{i+1}$. Let $Q$ be the partition defined by $Q(\omega)=\omega_{0}$. To construct the measure on $\Omega$ we will use a cutting and stacking approach. The 0 block consists of one 0 .

Like the Ornstein-Shields transformations, all $n$ blocks will have a random number of $f$ at the beginning of each block. In the middle of the $n$ block will be a large number, $N(n)$, of $n-1$ blocks seperated by a certain number of the spacer symbol $s$. All $n$ blocks end with a string of $e$.

All of the $n$ blocks will have the same length which we call $h(n)$. The number of $f$ at the beginning of an $n$ block, $F$, will be chosen with uniform distribution from $h(n-1)+1$ to $h(n-1)+n-1$. The number of $e$ at the end of an $n$ block is $2 h(n-1)+n-F$. This random number of $f$ at the beginning of the $n$ block will allow us to show that the transformation is $K$.

The number of the spacer symbols, $s$, between $n-1$ blocks in an $n$ block is determined in the following way. We will use numbers $x_{n}$ and $c_{n}$. We refer to the first $c_{n} x_{n} N(n) n-1$ blocks as the "cyclic" region of the $n$ block. The number of spacer symbols following each $n-1$ block in the cyclic region is determined by a sequence $p_{i, n}$. The first $x_{n} N(n) n-1$ blocks have $p_{1, n}$ 's following them. The next $x_{n} N(n) n-1$ blocks have $p_{2, n}$ 's following them. This continues all the way to the final $x_{n} N(n) n-1$ blocks in the cyclic region which have $p_{c_{n}, n}$ 's following them. The positioning of spacer symbols between $n$ blocks in the "cyclic" region will ensure that all the transformations we construct are loosely Bernoulli.

The rest of the $n-1$ blocks are in the "mixing" region of the $n$ block. The number of spacers between these $n-1$ blocks is determined by a "pseudorandom" sequence $a_{i, n}$ and a number $S(n)$. The "pseudorandom" sequence $a_{i, n}$ is determined the same way as it was in [13]. The first $n-1$ block in the mixing region has $a_{1, n}$ 's before it. (This is in addition to the $p_{c_{n}, n}$ following the last $n-1$ block in the cyclic region.) It also has $S(n)-a_{1, n}$ 's following it. The second $n-1$ block in the mixing region has $a_{2, n}$ 's before it. (Again, this is in addition to the $S(n)-a_{1, n}$ following the previous $n-1$ block.) It also has $S(n)-a_{2, n}$ 's following it. This same procedure determines the number of $s$ between all of the $n-1$ blocks in the mixing region of the $n$ block. The choice of the number of $s$ between $n-1$ blocks in the mixing region of the $n$ block will allow us to do the necessary coding arguments.

Corresponding to every choice of $F$ and every sequence of $N(n)(n-1)$ blocks is one $n$ block. Thus if there are $A(n-1)$ different $n-1$ blocks, there are $A(n)=$ $(A(n-1))^{N(n)}(n-1) n$ blocks all of length $h(n)$. We do the construction so that all of our $n$ blocks have equal measure.

The following is a picture of an $n$ block. The mixing region is much longer than the cyclic region. The $n-1$ blocks are much longer than the spacers before and 
after the $n-1$ blocks.

$$
\begin{aligned}
& \overbrace{f f f}^{F} \overbrace{\text { cyclic region }}^{\overbrace{(n-1 \text { block }) \ldots(n-1 \text { block })}^{x_{n} N(n)} \ldots(n-1 \text { block }) \overbrace{s s s s}^{p_{c_{n}, n}} \ldots(n-1 \text { block }) \overbrace{s s s s}^{p_{c_{n}, n}}} \\
& \underbrace{\overbrace{a_{1}, n}}_{\text {ssssssss }}(n-1 \text { block }) \overbrace{s s s s s s s s s}^{S(n)-a_{1, n}} \ldots \overbrace{\text { ssssss }}^{a_{\left(1-x_{n} c_{n}\right) N(n), n}}(n-1 \text { block }) \overbrace{\text { ssssssssssss }}^{S(n)-a_{\left(1-x_{n} c_{n}\right) N(n), n}} \\
& \text { mixing region } \\
& \overbrace{\text { eeeeeeeee }}^{2 h(n-1)+n-F}
\end{aligned}
$$

In order to complete our description we need to specify all the parameters listed above. Choose $x_{n}$ so that

$$
x_{n} \rightarrow 0 \quad \text { and } \quad \sum_{n=1}^{\infty}\left(x_{n}\right)^{p}=\infty \quad \text { for all } p .
$$

For technical reasons we choose $x_{n}=0$ if $n=0,2 \bmod 3$. Next we select $c_{n} \in \mathbf{Z}$ increasing to infinity, but doing so slowly enough that $c_{n} x_{n} \rightarrow 0$.

We will also need a sequence $\epsilon_{n}$ such that $\sum_{N}^{\infty} \epsilon_{n}<\epsilon_{N-1}$. To choose the sequence $p_{i, n}$ we will use a lemma in [3].

Lemma 2.1 ([3]). There exists a sequence $p_{1, n}, \ldots, p_{c_{n}, n}$ so that $h(n-1)+p_{1, n}$, $h(n-1)+p_{2, n}, \ldots, h(n-1)+p_{c_{n}, n}$ are relatively prime and $p_{i, n} / h(n-1)<\epsilon_{n}$ for all $i$.

As indicated in the picture above, we can always choose $p_{1, n}=0$.

We also need $S(n)$ so that $h(n-2) \ll S(n) \ll h(n-1)$ and a pseudorandom sequence, $a_{c_{n} x_{n}+1}, \ldots, a_{N(n)}$, of integers between 1 and $S(n)$. The pseudorandom sequence will be chosen in the same way as it was in [13]. We now introduce some notation which will let us describe the property that we want the sequence $a_{i, n}$ to have.

For $x \in \Omega$, we will say that a consecutive string of integers $i, \ldots, i+h(n)-1$ is an $n$ block in $x$ if $K^{j}(x)$ is in the same $n$ block for all $j, i \leq j \leq i+h(n-1)$. If $x, y \in \Omega$ we will say that an $n$ block in $x$ lines up with an $n$ block in $y$ line up if the two blocks of integers differ by at most $h(n-1)+S(n)$. We say that two points $x$ and $y$ have block structures that eventually line up if there exists an $N$ such that for all $n>N$ the $n$ block in $x$ containing 0 lines up with the $n$ block in $y$ containing 0 . For a collection of points $x_{1}, \ldots, x_{n}$ an $n$ mixing intersection is a maximal interval, $(i, j)$, such that $(i, j)$ is contained in the mixing region of an $n$ block for each $x_{k}$.

Lemma 2.2. There exists $N_{0}$ such that for $N(n)>N_{0}$ there is an appropriate "pseudorandom" sequence, $a_{1, n}, \ldots, a_{\left(1-x_{n} c_{n}\right) N(n), n}$ with the following property. For any points $\left(\omega_{1}, \omega_{2}, \ldots, \omega_{k}\right)$ any mixing intersection, $(i, j)$ of $n$ blocks, with $0 \leq k \leq n$, and consecutive strings of integers $Z_{1}, Z_{2}, \ldots, Z_{n} \subset(1, \ldots, h(m))$ $m \leq n-2$, one of three things must happen. Either

1) the mixing intersection is extremely short $\left(j-i<\epsilon_{n} h(n)\right)$,

2) there are two points $\omega_{t}$ and $\omega_{t^{\prime}}$ such that the $n$ blocks for $\omega_{t}$ and $\omega_{t^{\prime}}$ line up, or 
3) $\mid \frac{1}{j-i-1}$ (\# of $t \mid K^{t}\left(\omega_{1}\right)$ is in the $i_{1}$ th position of an $m$ block for some $i_{1} \in Z_{1}$, $K^{t}\left(\omega_{2}\right)$ is in the $i_{2}$ th position of an $m$ block for some $i_{2} \in Z_{2}, \ldots, K^{t}\left(\omega_{k}\right)$ is in the $i_{k}$ th position of an $m$ block for some $\left.i_{k} \in Z_{k}\right)-\prod\left(\left|Z_{i}\right| / h(n-2)\right) \mid$ $<\left(\prod\left(\left|Z_{i}\right|+n(n+1) / 2\right)-\prod\left|Z_{i}\right|\right) / h(n-2)^{k}+\epsilon_{n-2}$.

Proof. This follows immediately from a lemma in [13]. The only thing we have to account for is how the different choices of $f$ can affect the mixing properties of the pseudorandom sequence.

We have preserved the aspects of the construction of the Ornstein-Shields transformations which are used in the proof that they are $K$. Thus the proof that our basic transformation is a $K$ transformation is the same as the proof that the Ornstein-Shields transformations are $K$.

Lemma 2.3. $(K, \Omega, \mu)$ is a $K$ transformation.

Proof. We need to show that for any $k$, any $\epsilon>0$ and $\epsilon$ almost any past, $P$, which specifies the $Q$ name up to time 0 , that there exists an $M$ such that the conditional distribution given $P$ on $Q$ names during times $M$ to $M+k$ is within $10 \epsilon$ of the unconditional distribution of $Q$ names from times $M$ to $M+k$.

First pick an $n$ such that the measure of points in $n$ blocks is at least $1-\epsilon^{2} /(k+1)$. This tells us that the conditional distribution of strings of length $k+1$ which are entirely with an $n$ block is within $\epsilon^{2}$ of the unconditional distribution. Next choose $m$ such that $\epsilon(m-1)>h(n)$ and $M$ so that $M=h(m)$. Now $P$ may tell us whether $(M, M+k)$ is in an $m$ block, and where it is inside of an $m$ block, but it tells us nothing about which $m$ block it is in. Restrict to those pasts that tell us that with probability at least $1-\epsilon,(M, M+k)$ is inside of an $n$ block. This eliminates a set of pasts of measure less than $\epsilon$. Since we don't know the number of $f$ at the beginning of the $m$ block that $(M, M+k)$ is in, and $\epsilon(m-1)>h(n)$, we could be anywhere within an $n$ block with $\epsilon$ almost equal probability. Since we could be in any $n$ block with equal probability, independent of where we are in that $n$ block, the conditional distribution given $P$ is within $\epsilon$ of the conditional distribution given that we are in an $n$ block. This is within $\epsilon$ of the unconditional distribution on strings of length $k+1$.

Throughout this paper we are going to be working with the class of functions $S_{\pi}$ defined in the following way. Choose a countable or finite set $V$ and a permutation $\pi$ of $V$ that has only cycles of finite length. It causes no loss of generality to assume that cycles of $\pi$ are always consecutive integers. Define $S_{\pi}$ on $\Omega^{V}$ to be

$$
S_{\pi}\left(\omega_{1}, \omega_{2}, \ldots\right)=\left(K\left(\omega_{\pi(1)}\right), K\left(\omega_{\pi(2)}\right), \ldots\right) .
$$

Corollary 2.1. All the maps of the form $S_{\pi}$ are $K$.

Proof. We only need to prove it when $\pi$ is a $p$ cycle and $|V|=p$. Then we can use the fact that the direct product of $K$ automorphisms is a $K$ automorphism to prove the result. The only difference between this and the previous result, is that we want the probability that all $p$ points are in an $n$ block at least $1-\epsilon^{2}$ and we throw away pasts such that the conditional probability that all $p$ coordinates are in $n$ blocks is greater than $1-\epsilon$. Other than that, the proof goes through exactly.

Lemma 2.4. All of the transformations $S_{\pi}$ are loosely Bernoulli. 
Proof. The proof of this is similar to the proof of theorem 4 in [4]. In [5] it is shown how to construct a subset in a Bernoulli shift so that the induced transformation is $S_{\pi}$. Thus each $S_{\pi}$ is loosely Bernoulli.

\section{Properties of the Block structure}

An important property of the block structure is that if an $m$ block in $x$ intersects an $m$ block in $y$, but doesn't line up with it, then very few of the $n<m$ blocks in $x$ of the intersection will line up with an $n$ block in $y$. Choose $\beta_{m}$ to be a sequence approaching zero such that $2 \sup _{m>n} x_{m} c_{m} / \beta_{m}$ also approaches zero.

Definition 3.1. Define $l(n)$ to be the infimum over any two $m$ blocks, $m>n$, that intersect in a length at least $\beta_{m} h(m)$ but do not line up, of the fraction of the $n$ blocks in the intersection of the two $m$ blocks that do line up.

Lemma 3.1. $l(n) \rightarrow 0$ as $n \rightarrow \infty$.

Proof. Since the intersection is long enough, a fraction at least $1-2 \sup _{m>n} x_{m} c_{m} / \beta_{n}$ of it is a mixing intersection. Either $m-2$ or $m-3$ blocks have no "cyclic" section. By the Lemma 2.2, the fraction of the $m-2$ or $m-3$ blocks in the mixing portion of the $m$ intersection that do line up is at most $3 h(m-4) / h(m-3)$.

If $j$ blocks have no cyclic part and two $j$ blocks do not line up, then, by Lemma 2.2 almost all of the $j-i(3 \leq i \leq 5)$ blocks inside the intersection will not line up. The fraction of $j-i$ blocks that line up is less than $3 h(j-i-1) / h(j-i)$.

Choose $j$ to be $m-2$ or $m-3$ so that $j$ blocks do not have cyclic parts. By the first statement most of the $j$ blocks in the mixing part do not line up. Apply the second statement repeatedly with $i=3$ and then once with $i=3,4$, or 5 to arrive at $n$. Thus the fraction of $n$ blocks that line up is less than

$$
\begin{aligned}
l(n) & <2 \sup _{m>n}\left(2 x_{m} c_{m} / \beta_{m}+\sum_{n}^{m} 3 h(j-1) / h(j)\right) \\
& <2 \sup _{m>n} x_{m} c_{m} / \beta_{m}+\sum_{n}^{\infty} 3 h(j-1) / h(j) .
\end{aligned}
$$

Lemma 3.2. If none of the interval $(i, j)$ is in an $n$ block for a point $x$ and $|j-i|<$ $h(n)$, then the $x$ name from $i$ to $j$ can change only twice. That is there exists at most two $m, i \leq m \leq j$, such that $x_{m} \neq x_{m+1}$.

Proof. All strings of $f$ and $e$ that are not in an $n$ block are at least $h(n)$ long. Thus each such string of $f$ or $e$ must contain either $i$ or $j$ so there can be at most two of them. Since $(i, j)$ is not in an $n$ block there can be no 0 in the $\omega$ name from $i$ to $j$, so $s$ is the only other possible symbol. Thus there can be at most one string of $s$, $f$ or $e$.

Now we prove that the block structure of a point is well defined and meaningful. By this we mean that if we have $x, y \in \Omega$ and there is an $n$ block in $x$ which intersects, but does not line up with an $n$ block in $y$, then the $x$ and $y$ names disagree at least once in the intersection. This is proved in the following lemma. Lemma 3.4 tells us that we can make the percentage of disagreement independent of $n$.

The next two lemmas actually prove more than this. Under the same hypothesis we prove that for any $m<n$ the sequences $x_{m i+c}$ and $y_{m i+c}$ differ at least once 
in the overlap. This density of disagreement can also be made independent of $n$ (but not $m$ ). This will allow us to analyze the codes to coordinates which are being permuted with period $m$.

Lemma 3.3. For all $m$ and $n>m$ if $j, \ldots, j+h(n)-1$ is an $n$ block for $x \in \Omega$ and $x_{m i+c}=y_{m i+c}$ for all $i, j \leq m i+c \leq j+h(n)-1$, then there is an $n$ block of $y$ that lines up with the $n$ block of $x$.

Proof. If at least half of $j, \ldots, j+h(n)-1$ is not in an $n$ block for $y$, then $y$ can change only twice in this region. Any half of an $n$ block has many intervals of length at least $n$ of consecutive $f$ 's and $e$ 's. Thus on one of those intervals they must disagree. If most of $j, \ldots, j+h(n)-1$ is in an $n$ block which does not line up with the $n$ block for $x$, then, by Lemma 2.2, the $n-2$ blocks in the $n$ block intersections line up in nearly every possible way with about the same frequency. At least one of these ways has the at least $m$ consecutive $s$ of one $n-2$ block lining up with $m$ consecutive $f$ of another. For any $c$ there is at least one $i$ such that $x_{m i+c}=f$ and $y_{m i+c}=s$.

Now we show that points whose $n$ blocks intersect, but don't line up, not only disagree once on the $n$ intersection, they disagree at fixed percentage of the time, independent of $n$. This is also true if we look at names under time $m$.

Lemma 3.4. For all $m$ there exists an $N$ and an $\alpha_{m}>0$ such that for all $n>N$, $x, y \in \Omega, c$, and $j$ where $j, \ldots, j+h(n)-1$ is an $n$ block for $x$ we have the following. If $x_{m i+c}=y_{m i+c}$ for all but $\alpha_{m} h(n) / m$ of the $i, j \leq m i+c \leq j+h(n)-1$, then there is an $n$ block of $y$ that lines up with the $n$ block $j, \ldots, j+h(n)-1$ of $x$.

Proof. Choose $N$ such that $l(N)<1$ and $N>m$. If there is an $n>N$ block in $x$ which has no $n$ block in $y$ that lines up with it, then at most $1-l(N)$ of the $N$ blocks in the $n$ block for $x$ line up with an $N$ block for $y$. By the previous lemma if an $N$ block, $(j, j+h(N)-1)$, in $x$ doesn't line up with an $N$ block in $y$, then for every $c$ there exists an $i, j \leq m i+c \leq j+h(N)-1$ for $x$ where $x_{m i+c} \neq y_{m i+c}$. Thus for at least $(1-l(N))(1 / h(N))(\mu(N$ blocks $))=\alpha_{m}$ of the $i, j \leq m i+c \leq j+h(n)-1$ have $x_{m i+c} \neq y_{m i+c}$.

This last result is true even if we look at small portions of an $n$ block.

Lemma 3.5. If $j, \ldots, k$ is a large $\left(k-j>\beta_{n} h(n)\right)$ part of an $n, n>N, m$, block for $x$ and if $x_{m i+c}=y_{m i+c}$ for all but $\alpha_{m}(k-j) / 4 m$ of the $i$ such that $j \leq m i+c \leq k$, then there is an $n$ block of $y$ that lines up with the $n$ block of $x$ around $(j, \ldots, k)$.

Proof. If no $n$ block in $y$ lines up with the $n$ block around $(j, \ldots, k)$ in $x$, then by Lemma 2.2 and the fact that most of $(i, j)$ is a mixing intersection, at least $1 / 2$ of the $n-2$ blocks in $(j, \ldots, k)$ line up with no $n-2$ block in the $y$ name. Since the fraction of discrepancies is at least $\alpha_{m}$ in each of these $n-2$ blocks, and $n-2$ blocks make up greater than $1 / 2$ of $(j, \ldots, k)$, the fraction of differences must be at least $\alpha_{m} / 4$.

For some $n$ we need to see an even smaller number of symbols to identify where, in the $n$ block, the symbols came from.

Corollary 3.1. If $n \neq 1$ mod 3, then the previous lemma holds with $\epsilon_{n}$ instead of $\beta_{n}$.

Proof. If $n \neq 1 \bmod 3$, then there is no cyclic portion of $n$ blocks. The proof goes as above except now Lemma 2.2 applies to all of the intersection. 


\section{ISOMORPHISM OF THE TRANSFORMATIONS $S_{\pi}$}

Consider the transformations $S_{i d}\left(x_{1}, x_{2}\right)=\left(K x_{1}, K x_{2}\right)$ and $S_{(1,2)}\left(y_{1}, y_{2}\right)=$ $\left(K y_{2}, K y_{1}\right)$ on $\Omega \times \Omega$. In this section we will show that they are not isomorphic. The outline of the proof is as follows. Suppose $\Phi$ is an isomorphism of $S_{i d}(x, y)$ and $S_{(1,2)}(x, y)$ and $\Phi\left(x_{1}, x_{2}\right)=\left(y_{1}, y_{2}\right)$. Then either the block structure of $x_{1}$ eventually lines up with $y_{1}$ and the the block structure of $x_{2}$ eventually lines up with $y_{2}$ or the block structure of $x_{1}$ eventually lines up with $y_{2}$ and the the block structure of $x_{2}$ eventually lines up with $y_{1}$. Either way $\Phi$ and $\left(x_{1}, x_{2}\right)$ generate a permutation on two symbols. We show that this permutation must be independent of $\left(x_{1}, x_{2}\right)$. Thus it conjugates the permutations $i d$ and $(1,2)$. This is a contradiction. In general, we will prove that an isomorphism between $S_{\pi}$ and $S_{\pi^{\prime}}$ generates a permutation $\alpha$ such that $\pi^{\prime}=\alpha \pi \alpha^{-1}$. Thus $S_{\pi}$ and $S_{\pi^{\prime}}$ are isomorphic if and only if $\pi$ and $\pi^{\prime}$ are conjugate.

Now we will classify the isomorphisms of $S_{\pi}$ and $S_{\pi^{\prime}}$. If we have an isomorphism $\Phi$ of $S_{\pi}$ and $S_{\pi^{\prime}}$, then the code to each coordinate $i$, where $\pi^{\prime}(i)=i$, is a map of the form

$$
\Psi: \Omega \times \Omega \times \ldots \rightarrow \Omega \text { such that } \Psi S_{\pi}=K \Psi .
$$

For those coordinates $i$ where $i$ is in an $m$ cycle of $\pi^{\prime}$ there is a map of the form

$$
\Psi: \Omega \times \Omega \times \ldots \rightarrow \Omega \text { such that } \Psi\left(S_{\pi}\right)^{m}=K^{m} \Psi .
$$

We will study all possible maps of this second, more general form. If $\Psi$ codes to the $i$ th coordinate then there exists an $m$ associated with $\Psi$, which is the period of $i$ under $\pi^{\prime}$. Our goal in the next few lemmas is to show that if $\Psi\left(\omega_{1}, \omega_{2}, \ldots\right)=y$, then there exist $i$ such that, with probability 1 , the block structure of $\omega_{i}$ eventually lines up with the block structure of $y$. The collection of $\Psi$ generates a permutation associated with the isomorphism and allows us to prove that $\pi$ and $\pi^{\prime}$ are conjugate.

For any $\epsilon>0$, we can choose an $N$ large enough such that $(1, \ldots, N)$ is $\pi$ invariant and the map $\Psi$ can be approximated $\epsilon$ well by a finite code, $\Psi_{N}$, of length $N$. In addition, this code can be chosen to depend only on $\left(y_{1}, \ldots y_{N}\right)$.

Our first step is to show the $n$ block structure of the name that we code to depends almost exclusively on the $n$ intersections, and number of $f$ at the beginning of the $n$ blocks, of a finite number of names we are coding from. Thus it is nearly independent of the choice of $n-1$ blocks inside the $n$ intersection, and of the rest of the coordinates. This is the principle step needed to extend the counterexample machine from the zero entropy to $K$ transformations.

Definition 4.1. An $n$ overlap $\left(i_{1}, \ldots, i_{j}\right)$ of $\left(x_{1}, \ldots, x_{j}\right)$ means that for all $i_{k}$, $K^{i_{k}}\left(x_{1}\right)$ is in the first position of an $n$ block.

Lemma 4.1. There exists an $N$ and an $L$ such that for all $\epsilon>0$ and $n>L$ there exists a set $G, \mu(G)>1-\epsilon$ with the following property. Suppose $\left(y_{1}, y_{2}, \ldots\right)$, $\left(z_{1}, z_{2}, \ldots\right) \in G$, for all $i, 1 \leq i \leq N$, the $n$ block of $y_{i}$ around 0 lines up exactly with the $n$ block of $z_{i}$ around 0 , and the $n$ block of $y_{i}$ around 0 has the same number of $f$ as the $n$ block of $z_{i}$ around 0 . Then the $n$ blocks around 0 of $\Psi\left(y_{1}, y_{2}, \ldots\right)$ and $\Psi\left(z_{1}, z_{2}, \ldots\right)$ line up to within $6(h(n)+S(n))$.

Proof. Find an $N$ so that the code of length $N, \Psi_{N}$ is at least $\epsilon\left(\alpha_{m}\right)^{2} / 100$ good. Find an $L>N$ so that the ergodic theorem on the set of bad coding has kicked in $\epsilon\left(\alpha_{m}\right)^{2} / 100$ well by time $h(L)$, and almost every point in $\Omega$ has $\left(-3 \beta_{L} h(L), 3 \beta_{L} h(L)\right)$ 
in an $L$ block. Choose an $n>L$. We say a point $\left(z_{1}, z_{2}, \ldots\right)$ codes well under $\Psi_{N}$ if the ergodic theorem has kicked in $\epsilon\left(\alpha_{m}\right)^{2} / 100$ well for the set of bad coding and by time $h(L)$ and $\Psi\left(z_{1}, z_{2}, \ldots\right)$ has $\left(-3 \beta_{n} h(n), 3 \beta_{n} h(n)\right)$ in an $n$ block. Take two points, $\left(y_{1}, y_{2}, \ldots\right)$ and $\left(z_{1}, z_{2}, \ldots\right)$, that code well under $\Psi_{N}$, and for all i, $1 \leq i \leq N$, the $n$ blocks around 0 for $y_{i}$ and $z_{i}$ have the same number of $f$ at the beginning and line up exactly. We will show that for most choices of $\left(y_{1}, y_{2}, \ldots\right)$ and $\left(z_{1}, z_{2}, \ldots\right)$ the $n$ blocks of $\Psi\left(y_{1}, y_{2}, \ldots\right)$ and $\Psi\left(z_{1}, z_{2}, \ldots\right)$ line up to within $3(h(n-1)+S(n))$.

To do this we will find a new point $\left(w_{1}, w_{2}, \ldots\right)$ that has the same $n$ block overlap and $f$ as $\left(y_{1}, y_{2}, \ldots\right)$ and $\left(z_{1}, z_{2}, \ldots\right)$ and codes well under $\Psi_{N}$. Choose the $n-1$ blocks and $f$ of $\left(w_{1}, \ldots, w_{N}\right)$ so that the first third of the $n$ intersection around 0 is the same as the first third of the $n$ intersection around 0 of $\left(y_{1}, \ldots, y_{N}\right)$ and the last third is the same the as the intersection of $\left(z_{1}, \ldots, z_{N}\right)$. The set of points, $\left(y_{1}, y_{2}, \ldots\right)$ and $\left(z_{1}, z_{2}, \ldots\right)$, where we cannot find such a $\left(w_{1}, w_{2}, \ldots\right)$ that codes well under $\Psi_{N}$ is less than $\epsilon$.

Since the length of the code $\Psi_{N}$ is $N$, the first third of the intersection codes independently of the last third of the intersection. Each third codes $\alpha_{m} / 10$ well to a string of characters of length $>\beta_{n} h(n)$. Since the first and last thirds of $\left(w_{1}, w_{2}, \ldots\right)$ both code well, they must give a consistent location (to within $h(n-1)+S(n))$ for the $n$ block of $\Psi\left(w_{1}, w_{2}, \ldots\right)$. Since the first third of $\left(w_{1}, \ldots, w_{N}\right)$ is identical to the first third of $\left(y_{1}, \ldots, y_{N}\right) \Psi_{N}$ applied to the first third of the intersection of these two is identical. The last third of $\left(w_{1}, \ldots, w_{N}\right)$ is the same as $\left(z_{1}, \ldots, z_{N}\right)$. So $\Psi_{N}$ applied to the last third of the intersections of this pair is identical. Each such string determines the location of the $n$ block for $\Psi\left(y_{1}, y_{2}, \ldots\right)$ and $\Psi\left(z_{1}, z_{2}, \ldots\right)$ line up with $\Psi\left(w_{1}, w_{2}, \ldots\right)$ to within $(h(n-1)+S(n))$. Thus the $n$ blocks for $\Psi\left(y_{1}, y_{2}, \ldots\right)$ and $\Psi\left(z_{1}, z_{2}, \ldots\right)$ line up to within $3(h(n-1)+S(n))$.

Since this works for most $\left(z_{1}, z_{2}, \ldots\right)$ and $\left(y_{1}, y_{2}, \ldots\right)$, we can find a point $\left(x_{1}, x_{2}, \ldots\right)$ such that for most $\left(z_{1}, z_{2}, \ldots\right)$ that have $n$ overlap and $f$ the same as $\left(x_{1}, x_{2}, \ldots\right), \Psi\left(x_{1}, x_{2}, \ldots\right)$ and $\Psi\left(z_{1}, z_{2}, \ldots\right)$ line up to within $3(h(n-1)+S(n))$. If $\left(z_{1}, z_{2}, \ldots\right)$ and $\left(y_{1}, \ldots, y_{j}, \ldots\right)$ are in this good set, then $\Psi\left(z_{1}, z_{2}, \ldots\right)$ and $\Psi\left(y_{1}, y_{2}, \ldots\right)$ line up to within $6(h(n-1)+S(n))$. Let $G$ be the union of these good sets for every overlap and choice of $f$.

By looking at codes that are a little longer we see that not only do the $n$ block structures of the points we are coding from determine the $n$ block structure of the point we are coding to, the $n$ block around 0 of the point we are coding to lines up with the $n$ block around 0 of one of the points we are coding from.

Lemma 4.2. There exists $N$ and $L$ such that for any $n>L+2$ there exists a set $G, \mu(G)>1-\epsilon$, and $\left(y_{1}, y_{2}, \ldots\right) \in G$ and $\Psi\left(y_{1}, y_{2}, \ldots\right)=z$, then the $n$ block of $z$ must line up with an $n$ block of $y_{i}$ for some $i, 1 \leq i \leq N$.

Proof. Heuristically, we choose $N$ and $L$ as in the previous lemma and $G$ to be all points $\left(y_{1}, y_{2}, \ldots\right)$ that coded well and the $n$ blocks around 0 for $y_{1}, \ldots, y_{N}$ do not line up. If the $n$ block around 0 of $z=\Psi\left(y_{1}, y_{2}, \ldots\right)$ did not line up with the $n$ block around 0 of any of the $y_{1}, \ldots, y_{N}$, then, by Lemma 2.2 , for every different $n-2$ overlap of $y_{1}, \ldots, y_{N}$ we would see the $n-2$ blocks of $z$ in almost every possible position with nearly equal frequency. There are not enough possible choices of the $f$ so that it would be consistent with the previous lemma. 
We will prove this by contradiction. Choose a point $\left(y_{1}, y_{2}, \ldots\right)$ with $n$ intersection $(i, j)$ so that most of the $i \in(j, k)$ have $\left(S_{\pi}\right)^{i}\left(y_{1}, y_{2}, \ldots\right) \in G$ from the previous lemma, and none of the $n$ blocks of the $y_{i}, 1 \leq i \leq N$, line up. Choose any overlap of $N n-2$ blocks, $A$. By Lemma 2.2 in the $n$ intersection of $y_{1}, \ldots, y_{N}$, at the times we see the $n-2$ blocks, agree within $4 n$ with $A$; we see the $n-2$ block of $z$ in every position (within $6 h(n-3)+S(n-2)$ ) with almost equal frequency. There are $(8 n+1)^{N}(n-2)^{N}$ choices of close overlaps and $f$, but at least $N(n-2) / 6$ choices of the lining up (within $6 h(n-3)+S(n-2)$ ) of the $n-2$ block of $z$. Since the latter is growing exponentially, it could not be these intersections that agree with $A$ that uniquely determine the position of the $n-2$ block of $z$ (within $6 h(n-3)+S(n-2)$ ). But this holds for all $A$. Thus the $n-2$ block overlaps and $f$ of $\left(y_{1}, y_{2}, \ldots\right)$ cannot determine the position of most of the $n-2$ blocks of $z$. This is a contradiction.

Lemma 4.3. There exists an $i$ such that for almost all $\left(y_{1}, y_{2}, \ldots\right)$, the $n$ block of $\Psi\left(y_{1}, y_{2}, \ldots\right)$ around 0 lines up with the $n$ block around 0 of $y_{i}$ for all $n$ large enough.

Proof. Choose $n$ so that $l(n)<1 / 2 N$ and $n>L+2$, where $N$ and $L$ are from the previous lemma. The ergodic theorem and the previous lemma for $n$ imply that most of the $n$ blocks of $\Psi\left(y_{1}, y_{2}, \ldots\right)$ line up with an $n$ block of one of the $y_{i}$. We will now show that for $n$ large enough it is always the same $y_{i}$. Define $B_{i}$ to be the collection of points $\left(y_{1}, y_{2}, \ldots\right)$ that have the $n$ block around 0 of $y_{i}$ and the $n$ block around 0 of $\Psi\left(y_{1}, y_{2}, \ldots\right)$ lining up. By the previous lemma we know that $\sum_{1}^{N} \mu\left(B_{i}\right)>1 / 2$. Thus, for at least one $i$, we have $\mu\left(B_{i}\right)>1 / 2 N$. Now if $\left(y_{1}, y_{2}, \ldots\right)$ satisfies the ergodic theorem for the sets $B_{i}$, the long term averages of $\left(y_{1}, y_{2}, \ldots\right)$ are at least $1 / 2 N$ for some $B_{i}$. We now wish to show that for this $i$ the $n$ block structures of $\Psi\left(y_{1}, y_{2}\right)$ and $y_{i}$ eventually line up. If they did not, then there would be arbitrarily large $m$ such that the $m$ block around 0 of $\Psi\left(y_{1}, y_{2}, \ldots\right)$ and of $y_{i}$ didn't line up. Lemma 3.1 implies, that for $m>n$, less than $l(n)<1 / 2 N$ of the $n$ blocks in the $m$ intersection line up. This contradicts the fact that for all sufficiently long stretches at least $1 / 2 N$ of the $n$ blocks of $y_{i}$ and $\Psi\left(y_{1}, y_{2}, \ldots\right)$ line up. Thus any point, $\left(y_{1}, y_{2}, \ldots\right)$, that satisfies the ergodic theorem for the $B_{i}$ must code to a point that eventually lines up with $y_{i}$.

Theorem 4.1. $S_{\pi}$ and $S_{\pi^{\prime}}$ are isomorphic if and only if $\pi$ and $\pi^{\prime}$ are conjugate.

Proof. By the lemma above, if we are coding by $\Phi^{-1}$ from $S_{\pi^{\prime}}$ to $S_{\pi}, \Psi_{v}$ pairs up a coordinate $v$ with one coordinate, $\alpha(v)$, that we are coding from. Since for almost every point $\left(y_{1}, y_{2}, \ldots\right) \in \Omega^{V}$ none of the points $y_{i}$ and $y_{j}$ have block structures that eventually line up, we know that $\alpha$ is well defined. Since this works for every $v$, the map $\alpha$ is onto. We could also look at the map $\Phi$ and conclude that $\alpha$ is, in fact, one-to-one.

If $\Phi\left(y_{1}, y_{2}, \ldots\right)=\left(z_{1}, z_{2}, \ldots\right)$ and for all $i, y_{i}$ eventually lines up with $z_{\alpha(i)}$ applying $S_{\pi}$ and $S_{\pi^{\prime}}$, we see the point in the $\pi^{-1}(i)$ th coordinate eventually lines up with a point in $\left(\pi^{\prime}\right)^{-1}(\alpha(i))$ th coordinate. This shows that $\alpha(\pi)^{-1}=\left(\pi^{\prime}\right)^{-1} \alpha$ or $\pi^{\prime}=\alpha \pi \alpha^{-1}$. Thus $\pi$ and $\pi^{\prime}$ are conjugate. If we have a conjugation $\alpha, \pi^{\prime}=\alpha \pi \alpha^{-1}$, then define $\Phi\left(y_{1}, y_{2}, \ldots\right)=\left(z_{\alpha(1)}, z_{\alpha(2)}, \ldots\right)$. Now $\Phi S_{\pi}=S_{\pi^{\prime}} \Phi$.

\section{ISOMORPHISM OF FACTORS}

In this section we will work with a very special collection of factors which are generated by a group of permutations. A group of permutations $P \subset \operatorname{Sym}(V)$ 
generates a factor, $S_{\pi} \bmod P$, of $S_{\pi}$ if for all $p \in P$ there exists $p^{\prime} \in P$ such that $\pi p=p^{\prime} \pi$. This factor associates points $\left(y_{1}, y_{2}, \ldots\right)$ and $\left(y_{1}^{\prime}, y_{2}, \ldots\right)$ if $\left(y_{1}^{\prime}, y_{2}^{\prime}, \ldots\right)=$ $\left(y_{p(1)}, y_{p(2)}, \ldots\right)$ for some $p \in P$. We want to investigate when a factor generated by $P$ of a map $S_{\pi}$ is isomorphic to a factor generated by $P^{\prime}$ in a map $S_{\pi^{\prime}}$. We will deal only with the special case where $P$ is a group generated by permutations $p_{i}$, each of which has cycles of only one finite length, which act nontrivially only on disjoint subsets $V_{i}$ of $V$. Throughout the rest of the paper we will only deal with factors generated by permutation groups of this form.

If there exists an invertible map $\alpha: V \rightarrow V^{\prime}$ such that $\alpha(P)=P^{\prime}$ and $\alpha^{-1} \pi \alpha=$ $\pi^{\prime} \bmod P^{\prime}$, it is easy to construct an isomorphism between $S_{\pi} \bmod P$ and $S_{\pi^{\prime}}$ $\bmod P^{\prime}$. The existence of such an $\alpha$ was a necessary condition in [13], and is almost certainly a necessary condition for us as well. However, in this section we prove a slightly weaker necessary condition. It should not be too difficult to get the necessary and sufficient condition, or to work with more general permutation groups; but we do not, because we do not need to for any of the examples we want to construct and it would even further complicate the notation.

For example, consider the transformation $S_{i d}$ on $\Omega^{4}$. Define $P_{1}$ to be the subgroup of $\mathrm{Sym}_{4}$ generated by the permutation $(1,2)(3,4)$ and $P_{2}$ to be the subgroup generated by the two permutations $(1,2)$ and $(3,4)$. We will show that no two of $S_{i d}, S_{i d} \bmod P_{1}$, and $S_{i d} \bmod P_{2}$ are isomorphic.

If $p$ is an $n$ cycle on $(1, \ldots, n)$, define $\Omega_{p}^{n}$ to be the space obtained by associating a point $\left(\omega_{1}, \ldots, \omega_{n}\right)$ with the points of the form $\left(\omega_{p^{m}(1)}, \ldots, \omega_{p^{m}(n)}\right)$. We also define a partition, $Q_{p}^{n}$, on $\Omega_{p}^{n}$. Given an $n$ cycle $p \in \operatorname{Sym}(1, \ldots, n)$ we associate $q=\left(q_{1}, q_{2}, \ldots, q_{n}\right)$ and $q^{\prime}=\left(q_{1}^{\prime}, q_{2}^{\prime}, \ldots, q_{n}^{\prime}\right)$ (all $q_{i}, q_{i}^{\prime} \in Q$ ) if for some $m$ and all $i$, $q_{i}=q_{p^{m}(i)}^{\prime} \cdot Q_{p}^{n}\left(y_{1}, \ldots, y_{n}\right)$ is the element of $Q_{p}^{n}$ that $\left(y_{1}, \ldots, y_{n}\right)$ is in.

Definition 5.1. Two coordinates $i$ and $j$ are associated under the factor generated by $P$ if there exists a $p \in P$ such that $p(i)=j$.

This divides $V$, the set of coordinates into equivalence classes. In the previous section we took an isomorphism and saw how it coded to each coordinate. Now we will look at how our isomorphism codes to a set of coordinates associated by $P^{\prime}$. If $1, \ldots, n \subset V^{\prime}$ are associated by an $n$ cycle $p^{\prime} \in P^{\prime}$ and $\left.\left(\pi^{\prime}\right)^{m}\right|_{(1, \ldots, n)}=i d$, then any isomorphism $\Phi$ between $S_{\pi} \bmod P$ and $S_{\pi^{\prime}} \bmod P^{\prime}$ gives us a shift invariant map

$$
\Psi: \Omega \times \ldots \times \Omega \times \ldots \rightarrow \Omega_{p}^{n} \text { such that } \Psi\left(S_{\pi} \bmod P\right)^{m}=\left(S_{\left.\pi^{\prime}\right|_{(1, \ldots, n)}} \bmod p^{\prime}\right)^{m} \Psi .
$$

To classify the possible isomorphisms $\Phi$ we will study the possible $\Psi$.

By choosing a large enough $N$ for this map, $\Psi$ can be approximated arbitrarily well by a map

$$
\Psi_{N}: \Omega \times \ldots \times \Omega \times \ldots \rightarrow Q_{p}^{s}
$$

which depends only on coordinates $(1, \ldots, N)$ at times $(-N, N)$. Our first goal is to show that if $\Psi\left(\omega_{1}, \omega_{2}, \ldots\right)=\left(y_{1}, \ldots, y_{n}\right) \bmod p$, then for all $i, y_{i}$ lines up with some $\omega_{j}$.

This analysis is not really very different from what we did in the previous section, but the notation is more complicated. The first lemma in this section is new, but Lemmas 5.2 through 5.5 are similar to lemmas in the previous sections.

First we need an analog of Lemma 3.3 that says, if we have two points, $x$ and $y$, and an $n$ block for $x$ does not line up with an $n$ block for $y$, then there exists a disagreement in $x$ and the $y$ name in the intersection. It also says the same thing 
about names under time $n$. This next lemma says that if we have two sets of points, $\left(x_{1}, \ldots, x_{s}\right)$ and $\left(y_{1}, \ldots, y_{s}\right)$, then either for every $i$ there is a $j$ such that the $n$ block around 0 of $x_{i}$ lines up with the $n$ block around 0 for $y_{j}$, or the $Q_{p}^{s}$ names of $\left(x_{1}, \ldots, x_{s}\right)$ and $\left(y_{1}, \ldots, y_{s}\right)$ disagree at least once on the $n$ intersection around 0 . The same thing holds for names under time $m$.

Lemma 5.1. Choose $s, m, 10<n, 0 \leq c<m,\left(x_{1}, \ldots, x_{s}\right)$, and $(j, k)$ a long enough $\left(k-j>\beta_{n^{2}} h\left(n^{2}\right)\right) n^{2}$ intersection of $\left(x_{1}, \ldots, x_{s}\right)$. If there exists $\left(y_{1}, \ldots, y_{s}\right)$ such that for all $r: j \leq m r+c \leq k$,

$$
Q_{p}^{s}\left(\left(S_{\pi}\right)^{m r+c}\left(y_{1}, \ldots, y_{s}\right)\right)=Q_{p}^{s}\left(\left(S_{\pi}\right)^{m r+c}\left(x_{1}, \ldots, x_{s}\right)\right),
$$

then for each $x_{i}$ there is a $y_{j}$ such that the $n$ block around $(j, k)$ of $y_{j}$ lines up with the $n$ block of $x_{i}$.

Proof. There are 3 cases that we must deal with. The first two assume that at least three quarters of $(j, k)$ is contained in an $n^{2}$ block for each $y_{i}$. The first case is that no two $n^{2}$ blocks for the $y_{k}$ line up to within $h\left(n^{2}-3 s\right)$. If the lemma is not true, then there exists $x_{a}$ such that the $n^{2}$ block for $x_{a}$ does not line up to within $h\left(n^{2}-3 s\right)$ of any of the $n^{2}$ blocks for the $y_{k}$ either. By Lemma 2.2, if none of the $n^{2}$ blocks for $x_{a}$ and $y_{k}$ line up to within $h\left(n^{2}-3 s\right)$, then all possible $n^{2}-3 s-2$ block overlaps are almost equally likely. Thus there is one overlap where all the $n^{2}-3 s-2$ blocks from $y$ s are all in the middle of $s$ and $x_{a}$ is in $f$ in the beginning an $n^{2}-3 s-2$ block. Then there exists a $t$ such that for all $v \in(t, t+n)$ and $b$ $\left(y_{b}\right)_{v}=s$ and $\left(y_{a}\right)_{v}=f$.

The second case is that there exists some $q, n^{2}-3 s \leq q<n^{2}$ such that for any pair, $y_{b}$ and $y_{c}$, the $n^{2}$ blocks either don't line up to within $h(q)$, or they do line up to within $h(q-3)$. Again, we will argue by contradiction. Take a maximal set of $b$ so that all the $n^{2}$ blocks corresponding to $y_{b}$ and $x_{a}$ don't line up to within $h(q)$. Then, inside their $q+1$ intersection, their $q-1$ blocks line up in all possible ways. In particular, there is one $q-1$ overlap where all the $y_{b}$ are in the middle of the $s$ between $q-2$ blocks and the $x_{a}$ is in $f$ starting the $q-1$ block. The other $y_{b}$ have their $n^{2}$ blocks differ from the one in our maximal set by less than $h(q-3)$ and their $q$ blocks differ by less than $h(q-3)+\sum_{q-3}^{n^{2}} f(i)$. Since that is much smaller than the number of $s$, they too are in the middle of the $s$ between $q-2$ blocks. Thus there exists a $t$ such that for all $v \in(t, t+n)$ and $b\left(y_{b}\right)_{v}=s$ and $\left(y_{a}\right)_{v}=f$.

The third case is that a quarter of $(j, k)$ is not in an $n^{2}$ block for one or more $y_{b}$. Over this long stretch the $y_{b}$ name can change at most twice. By applying the arguments we used on the $y_{i}$ above we can find many stretches of time where the $x_{i}$ names are $f$ or are all $s$. Thus there must be one stretch where the $y_{b}$ disagrees with all of the $x_{i}$ names.

The next lemma shows the density of disagreement is independent of $n$.

Lemma 5.2. There exists an $N$ and an $\alpha_{m}>0$ such that if $i, \ldots, j$ is a long enough $\left(j-i>\beta_{n}\right)$ portion of an $n, n>s^{2}, N$ block intersection for $\left(x_{1}, \ldots, x_{s}\right)$ and $Q_{p}^{n}\left(\left(S_{\pi}\right)^{m k+c}\left(x_{1}, \ldots, x_{s}\right)\right)=Q_{p}^{n}\left(\left(S_{\pi}\right)^{m k+c}\left(y_{1}, \ldots, y_{s}\right)\right)$ for all but $\alpha_{m}(j-i) / m$ of the $k, i \leq m k+c \leq j$, then there exist $n$ blocks of $\left(y_{1}, \ldots, y_{s}\right)$ which line up with the $n$ blocks of $\left(x_{1}, \ldots, x_{s}\right)$ around $(i, \ldots, j)$.

Proof. Choose $N$ such that $l(N)<1 / s$. If an $m$ block around 0 for $x_{i}$ doesn't line up with any $m$ block around 0 for $\left(y_{1}, \ldots, y_{s}\right)$, then in the intersection at most 
$\operatorname{sl}(N)$ of the $N$ blocks for the $x_{i}$ line up with no $N$ block for a $y_{j}$. By the previous lemma, on each of those $N$ blocks there must be at least 1 disagreement. At least

$$
(1-s l(N))(1 / h(N))(\mu(N \text { blocks }))=\alpha_{m}
$$

of the $k, i \leq m k+c \leq j$ have

$$
Q_{p}^{n}\left(S_{\pi}^{m k+c}\left(x_{1}, \ldots, x_{s}\right)\right)=Q_{p}^{n}\left(S_{\pi}^{m k+c}\left(y_{1}, \ldots, y_{s}\right)\right) .
$$

Thus the density of disagreement is independent of $n$.

Corollary 5.1. If $n \neq 1 \bmod 3$, then the previous lemma holds with $\epsilon_{n}$ instead of $\beta_{n}$.

Proof. If $n \neq 1 \bmod 3$, then the entire intersection is a mixing intersection.

We now proceed as we did in the previous section to show that the block structure of a coded cycle depends only on the block structure of the original names, not how they were filled in. In this lemma we show that if $\Psi$ codes from $\left(y_{1}, y_{2}, \ldots\right)$ to $\left(x_{1}, \ldots, x_{n}\right) \bmod p$, then each $x_{j}$ eventually lines up with some $y_{i}$.

Lemma 5.3. There exists a set $G, \mu(G)>1-\epsilon$, such that for all $\left(y_{1}, y_{2}, \ldots\right)$, and $\left(z_{1}, z_{2}, \ldots\right) \in G$ where the $n$ blocks $1 \leq i \leq N$ of $y_{i}$ and $z_{i}$ around 0 line up exactly and have the same number of $f$ at the beginning, then if $\Psi\left(y_{1}, y_{2}, \ldots\right)$ $=\left(y_{1}^{\prime}, \ldots, y_{n}^{\prime}\right) \bmod p$ and $\Psi\left(z_{1}, z_{2}, \ldots\right)=\left(z_{1}^{\prime}, \ldots, z_{n}^{\prime}\right) \bmod p$ for every $n$ block around 0 of a $y_{i}^{\prime}$, there exists an $n$ block of a $z_{j}^{\prime}$ that lines up with it to within $6(h(n-1)+S(n))$.

Proof. Find an $N$ so that the code of length $N, \Psi_{N}$ is at least $\epsilon\left(\alpha_{m}\right)^{2} / 100$ good. Find an $L>N$ so that the ergodic theorem on the set of bad coding has kicked in $\epsilon\left(\alpha_{m}\right)^{2} / 100$ well by time $h(L)$ and almost every point has $\left(-3 \beta_{L} h(L), 3 \beta_{L} h(L)\right)$ in an $L$ intersection. Choose an $n>L$. We say a point $\left(z_{1}, z_{2}, \ldots\right)$, codes well under $\Psi_{N}$ if the ergodic theorem has kicked in $\epsilon\left(\alpha_{m}\right)^{2} / 100$ well by time $h(L)$ and $\Psi_{N}\left(z_{1}, z_{2}, \ldots\right)$ has $\left(-3 \beta_{n} h(n), 3 \beta_{n} h(n)\right)$ in an $n$ intersection. If two sets of points, $\left(y_{1}, y_{2}, \ldots\right)$ and $\left(z_{1}, z_{2}, \ldots\right)$, code well and have the same $n$ block overlap and $f$, but are filled in differently, we will show that for most $\left(y_{1}, y_{2}, \ldots\right)$ and $\left(z_{1}, z_{2}, \ldots\right)$ the $n$ overlaps of $\Psi\left(y_{1}, y_{2}, \ldots\right)$ and $\Psi\left(z_{1}, z_{2}, \ldots\right)$ line up to within $3(h(n-1)+S(n))$.

To do this we will find $\Psi_{N}$ and a point $\left(w_{1}, w_{2}, \ldots\right)$ that has the same $n$ block overlap and $f$ as $\left(y_{1}, y_{2}, \ldots\right)$ and $\left(z_{1}, z_{2}, \ldots\right)$ and codes well under $\Psi_{N}$. Choose the $f$ and $n-1$ blocks inside the $n$ intersection of $\left(w_{1}, \ldots, w_{N}\right)$ so that the first third is the same as the $n-1$ blocks inside the $n$ intersection of $\left(y_{1}, \ldots, y_{N}\right)$ and the last third is the same as $\left(z_{1}, \ldots, z_{N}\right)$.

Now consider $\Psi_{N}\left(z_{1}, z_{2}, \ldots\right)$. Since the length of the code is $N$, the first third of the name codes independently of the last third of the name. Both the first and last thirds code well, so each third gives a consistent location (to within $h(n-1)+S(n)$ ) for the $n$ overlap. Since the first third of $\left(w_{1}, w_{2}, \ldots\right)$ is identical to the first third of $\left(y_{1}, y_{2}, \ldots\right) \Psi_{N}$ applied to the first third of the intersection of these two is identical. The last third of $\left(w_{1}, w_{2}, \ldots\right)$ is the same as the last third of $\left(z_{1}, z_{2}, \ldots\right)$, so $\Psi_{N}$ applied to the last third of the intersections of this pair is identical. Each third codes $\epsilon$ well to a string of characters of length $>\beta_{n} h(n)$. Each such string determines the location of the $n$ overlap to within $h(n-1)+S(n)$. Thus for most $\left(z_{1}, z_{2}, \ldots\right)$ and $\left(y_{1}, y_{2}, \ldots\right)$ that both code well, they code to strings that are in the same place inside an $n$ block to within $3(h(n-1)+S(n))$. 
Since this works for most $\left(z_{1}, z_{2}, \ldots\right)$ and $\left(y_{1}, y_{2}, \ldots\right)$, we can find a point $\left(x_{1}, x_{2}, \ldots\right)$ such that for most $\left(z_{1}, z_{2}, \ldots\right)$ that have $n$ overlap and $f$ the same as $\left(x_{1}, x_{2}, \ldots\right)$, the $n$ block overlaps of $\Psi\left(x_{1}, x_{2}, \ldots\right)$ and $\Psi\left(z_{1}, z_{2}, \ldots\right)$ line up to within $3(h(n-1)+S(n))$. If $\left(z_{1}, z_{2}, \ldots\right)$ and $\left(y_{1}, y_{2}, \ldots\right)$ are in this good set, then $n$ block overlaps of $\Psi\left(z_{1}, z_{2}, \ldots\right)$ and $\Psi\left(y_{1}, y_{2}, \ldots\right)$ line up to within $6(h(n-1)+S(n))$. Let $G$ be the union of these good sets for every overlap and choice of $f$.

Lemma 5.4. There exists an $N$ and an $L$ such that for all $n>L$, there is a set $G, \mu(G)>1-\epsilon$, such that $\left(y_{1}, y_{2}, \ldots\right) \in G$ and $\Psi\left(y_{1}, y_{2}, \ldots\right)=\left(z_{1}, \ldots, z_{s}\right) \bmod$ $p$ imply that there exist $i_{1}, \ldots, i_{s}$ such that the $n$ block overlaps of $\left(z_{1}, \ldots, z_{s}\right)$ and $\left(y_{i_{1}}, \ldots, y_{i_{s}}\right)$ around 0 line up.

Proof. Suppose not. Then there exists a $z_{i}$ that does not line with any of the $y_{i}$. Choose a point so that for most $t$ in the $n$ intersection $\left(S_{\pi}\right)^{t}\left(y_{1}, y_{2}, \ldots\right)$ are in $G$ from the previous lemma and none of the $n$ blocks of $y_{i}, 1 \leq i \leq N$ line up. Choose any overlap of $N n-2$ blocks, $A$. By Lemma 2.2 in the $n$ intersection of $y_{1}, \ldots, y_{N}$ at the times, we see all the $n-2$ overlaps that agree within $4 n$; with $A$ we see the $n-2$ block of $z_{i}$ in every position within $6(h(n-3)+S(n-2))$ with almost equal frequency. There are $(8 n+1)^{N}(n-2)^{N}$ choices of close overlaps and $f$, but there are at least $N(n-2) / 6$ choices of the lining up within $6(h(n-3)+S(n-2))$ of the $n-2$ block of $z_{i}$. Since the latter grows exponentially, it could not be these overlaps close to $A$ that uniquely determine the position of the $n-2$ block of $z$ within $6(h(n-3)+S(n-2))$. But this holds for all $A$. Thus the $n-2$ block overlaps and $f$ of $\left(y_{1}, y_{2}, \ldots\right)$ cannot determine the position of most of the $n-2$ blocks of $z$.

Lemma 5.5. There exists $i_{1}, \ldots, i_{s}$, so for almost every point $\left(y_{1}, y_{2}, \ldots\right)$, if $\Psi\left(y_{1}, y_{2}, \ldots\right)=\left(z_{1}, \ldots, z_{s}\right)$ mod $p$, then the $n$ block overlaps of $\left(z_{1}, \ldots, z_{s}\right)$ around 0 line up with the $n$ block overlaps of the $\left(y_{i_{1}}, \ldots, y_{i_{s}}\right)$ around 0 for all $n$ large enough.

Proof. Choose $n$ so that $l(n)<\frac{1}{2 N^{s}}$. By the previous lemma there exist $1 \leq$ $i_{1}, \ldots, i_{s} \leq N$ that have almost all of the $n$ block overlaps of $\left(z_{1}, \ldots, z_{s}\right)$ line up with an $n$ block overlap of $y_{i_{1}}, \ldots, y_{i_{s}}$. We will now show that for $n$ large enough it is always the same $y_{i}, \ldots, y_{i_{s}}$. Call the points in the collection of overlaps of $n$ blocks that have the $n$ block of $y_{i}, \ldots, y_{i_{s}}$ and the $n$ block of $\Psi\left(y_{1}, y_{2}\right)$ lining up $B_{i_{1}, \ldots, i_{s}}$. By the previous lemma $\sum \mu\left(B_{i_{1}, \ldots, i_{s}}\right)>1 / 2$. Now if $\left(y_{1}, y_{2}, \ldots\right)$ satisfies the ergodic theorem for the sets $B_{i_{1}, \ldots, i_{s}}$, the long term averages of $\left(y_{1}, y_{2}, \ldots\right)$ are at least $\frac{1}{2 N^{s}}$ for some $B_{i_{1}, \ldots, i_{s}}$. We now wish to show that for this $i_{1}, \ldots, i_{s}$, the block structures bigger than $n$ of $\Psi\left(y_{1}, y_{2}, \ldots\right)$ and $\left(y_{i_{1}}, \ldots, y_{i_{s}}\right)$ line up. If they didn't, then there would be some $m$ and $j$ such that the $m$ block around 0 of $z_{j}$ lines up with none of the $m$ blocks around 0 for $\left(y_{i_{1}}, \ldots, y_{i_{s}}\right)$. By Lemma 3.1 this implies that less than $l(n)<\frac{1}{2 N^{s}}$ of the $n$ blocks for $z_{j}$ inside the $m$ intersection line up with an $n$ block for some $y_{i_{k}}$. This contradicts the fact that for all sufficiently long stretches at least $\frac{1}{2 N^{s}}$ of the $n$ overlaps line up. Thus any points that satisfies the ergodic theorem for the $B_{i_{1}, \ldots, i_{s}}$ must code to a point that eventually lines up with $y_{1_{1}}, \ldots, y_{i_{s}}$.

As we said in the beginning of this section, if $P_{1}$ is generated by $(1,2)(3,4)$, we want to show that $S_{i d}$ and $S_{i d} \bmod P_{1}$ are not isomorphic. This next lemma will do just that. 
Lemma 5.6. If we have an isomorphism $\Phi$ from $S_{\pi} \bmod P$ to $S_{\pi^{\prime}} \bmod P^{\prime}$, the coordinates $(1, \ldots, n)$ are associated by $P$, and they line up with coordinates $i_{1}, \ldots, i_{n}$, then $i_{1}, \ldots, i_{n}$ must be associated.

Proof. Suppose not. Then there exists a minimal $P^{\prime}$ invariant subset $S^{\prime}$ such that $S^{\prime} \cap\left(i_{1}, \ldots, i_{n}\right) \neq \emptyset$ and $\left(S^{\prime}\right)^{C} \cap\left(i_{1}, \ldots, i_{n}\right) \neq \emptyset$. Suppose

$$
\Phi\left(\left(x_{1}, x_{2}, \ldots\right) \bmod P\right)=\left(\left(y_{1}, y_{2}, \ldots\right) \bmod P^{\prime}\right) .
$$

We want to look at $\Psi$

$$
\left(x_{1}, x_{2}, \ldots\right) \rightarrow\left(x_{1}, x_{2}, \ldots\right) \bmod P \rightarrow\left(y_{i}, \ldots\right)\left(i \in S^{\prime}\right) \bmod P^{\prime} .
$$

From Lemma 5.5 we know that there is a set $S$ of $\left|S^{\prime}\right|$ coordinates such that for every $j \in S, x_{j}$ lines up with some $y_{i}, i \in S^{\prime}$ for almost all points $\left(x_{1}, x_{2}, \ldots\right)$. Because $S^{\prime} \cap\left(i_{1}, \ldots, i_{n}\right) \neq \emptyset$, we have that $S \cap(1, \ldots, n) \neq \emptyset$ and because $\left(S^{\prime}\right)^{C} \cap$ $\left(i_{1}, \ldots, i_{n}\right) \neq \emptyset$, we have that $S^{C} \cap(1, \ldots, n) \neq \emptyset$.

Now take a permutation $p \in P$ which contains the cycle $(1, \ldots, n)$. Since $S \neq(1, \ldots, n)$, the points $x_{p(i)}, i \in S$, don't all line up with one of the points $y_{i_{1}}, \ldots, y_{i_{n}}$. On the other hand,

$$
\left(x_{p(1)}, x_{p(2)}, \ldots\right) \rightarrow\left(x_{1}, x_{2}, \ldots\right) \bmod P \rightarrow\left(y_{1}, y_{2}, \ldots\right) \bmod P^{\prime} .
$$

So the points $x_{p(i)}, i \in S$, should all line up with one of the points $y_{i_{1}}, \ldots, y_{i_{n}}$. This is a contradiction.

As we said at the beginning of the section, we want to show $S_{i d} \bmod P_{1}$, and $S_{i d} \bmod P_{2}$ are not isomorphic, where $P_{1}$ is the subgroup generated by the permutation $(1,2)(3,4)$ and $P_{2}$ is the subgroup generated by the two permutations $(1,2)$ and $(3,4)$. The next lemma will do that.

Definition 5.2. We say that two cycles $\left(i_{1}, \ldots, i_{n}\right)$ and $\left(j_{1}, \ldots, j_{n}\right)$ are linked under $P$ if for all $p \in P, p\left(i_{1}\right)=i_{2}$ implies $p\left(j_{1}\right)=j_{2}$.

Now we show that isomorphisms take linked cycles to linked cycles.

Lemma 5.7. If $\Phi$ is an isomorphism between $S_{\pi} \bmod P$ and $S_{\pi^{\prime}} \bmod P^{\prime}$ such that $\left(i_{1}, \ldots, i_{n}\right)$ and $\left(j_{1}, \ldots, j_{n}\right)$ are linked under $P^{\prime}$ and they line up with the cycles $(1, \ldots, n)$ and $(n+1, \ldots, 2 n)$, then $(1, \ldots, n)$ and $(n+1, \ldots, 2 n)$ are linked under $P$.

Proof. We have $\Phi^{\prime}$, the factor map composed with the isomorphism

$$
\Phi^{\prime}\left(x_{1}, x_{2}, \ldots\right) \rightarrow\left(x_{1}, x_{2}, \ldots\right) \bmod P \rightarrow\left(y_{1}, y_{2}, \ldots\right) \bmod P^{\prime} .
$$

We define "Pairings" to be all possible ways to divide $i_{1}, \ldots i_{n}, j_{1}, \ldots, j_{n}$ into pairs so no pair is of the form $\left(i_{k}, i_{m}\right)$ or $\left(j_{k}, j_{m}\right)$. Define a function from

$$
F: \Omega \times \Omega \times \ldots \rightarrow \text { "Pairings" }
$$

in the following way. Take $\left(x_{1}, x_{2}, \ldots\right)$ and find $\left(y_{1}, y_{2}, \ldots\right)$ such that

$$
\Phi^{\prime}\left(x_{1}, x_{2}, \ldots\right)=\left(y_{1}, y_{2}, \ldots\right) \bmod P .
$$

For each $k, 1 \leq k \leq n$, write down the pair of coordinates that line up with $i_{k}$ and $j_{k}$. Call those $n$ pairs $F\left(x_{1}, x_{2}, \ldots\right)$. This is well defined after we have made our choice of $i_{1}$ and $j_{1}$ because the cycles $\left(i_{1}, \ldots, i_{n}\right)$ and $\left(j_{1}, \ldots, j_{n}\right)$ are linked under $P^{\prime}$. 
Pick $k$ so that $\pi^{k}=\left(\pi^{\prime}\right)^{k}=i d$ when restricted to the coordinates $(1, \ldots, 2 n)$ and $\left(i_{1}, \ldots, i_{n}, j_{1}, \ldots j_{n}\right)$, respectively. $F$ is $\left(S_{\pi^{\prime}}\right)^{k}$ invariant because

$$
\Phi^{\prime}\left(S_{\pi^{\prime}}\right)^{k}\left(x_{1}, x_{2}, \ldots\right)=\left(S_{\pi}\right)^{k}\left(y_{1}, y_{2}, \ldots\right) \bmod P
$$

and these points generate the same pairings. Thus we have that $F$ is a shift invariant function into a finite set. Thus $F$ is constant a.e.

Now suppose $(1, \ldots, n)$ and $(n+1, \ldots, 2 n)$ are not linked. Then there would exist a $p$ such that $p(1)=2$ and $p(n+1)=n+1$. But

$$
\left(x_{p(1)}, x_{p(2)}, \ldots, x_{p(n+1)}, \ldots\right)=\left(x_{2}, \ldots, x_{n+1}, \ldots\right) \text { and }\left(y_{1}, y_{2}, \ldots\right)
$$

give us different pairings than $\left(x_{1}, x_{2}, \ldots, x_{n+1}, \ldots\right)$ and $\left(y_{1}, y_{2}, \ldots\right)$. This shows that $F\left(x_{p(1)}, x_{p(2)}, \ldots\right) \neq F\left(x_{1}, x_{2}, \ldots\right)$, which is a contradiction.

Thus an isomorphism takes $n$ cycles to $n$ cycles and linked $n$ cycles to linked $n$ cycles. We summarize our results in the following theorem. It applies only to $P$ and $P^{\prime}$ of the form we described at the beginning of the section.

Theorem 5.1. If $S_{\pi} \bmod P$ and $S_{\pi^{\prime}} \bmod P^{\prime}$ are isomorphic, then $P$ is conjugate to $P^{\prime}$. Also, restricted to the unlinked coordinates, we must have $\pi$ conjugate to $\pi^{\prime}$. $A$ sufficient condition is that there exist an $\alpha: V \rightarrow V^{\prime}$ such that $\alpha P \alpha^{-1}=P^{\prime}$ and $\alpha \pi \alpha^{-1}=\pi^{\prime} \bmod P^{\prime}$.

Proof. The necessary condition is a summary of Theorem 4.1 and Lemmas 5.6 and 5.7. The sufficient condition comes from the map that takes a point $\left(x_{1}, x_{2}, \ldots\right)$ $\bmod P$ to $\left(x_{\alpha(1)}, x_{\alpha(2)}, \ldots\right) \bmod P^{\prime}$.

\section{EXAMPLES}

All of the examples in this section are analogs of zero entropy transformations created by Rudolph in [13]. Ornstein's isomorphism theorem shows that it is impossible to have Bernoulli shifts like the following examples [7]. All of the examples below are of infinite entropy. In the next section we will show how to turn them into finite entropy examples.

Example 6.1. There exists an uncountable family of nonisomorphic infinite entropy $K$ transformations.

For finite entropy an example of this was first created by Ornstein and Shields [10]. Call the $j$ th prime $a_{j}$. For each sequence $x_{i}$ of 0's and 1's choose a permutation $\pi_{x}$ of $V=\mathbf{Z}$ that has a cycle of length $a_{j}$ if and only if $a_{j}=1$. Any two different sequences will generate permutations which are not conjugate. Thus the transformations generated by these permutations are not isomorphic.

Example 6.2. There exist two $K$ transformations which are not isomorphic but are isomorphic for all times $n \neq 1,-1$.

This was first done by Rudolph in [11]. Take $\pi$ to be a permutation of $V=\mathbf{Z}$ such that for every $j$, which is the product of an odd number of primes, there are infinitely many cycles of length $j$. In addition, we demand that there are no cycles of length $k$, where $k$ is the product of an even number of primes. Take $\pi^{\prime}$ to be a permutation of $V^{\prime}=\mathbf{Z}$ such that for every $k$ which is the product of an even number of primes, and there are infinitely many cycles of length $k$ and there are no cycles with length $j$ where $j$ is the product of an odd number of primes. Since these two permutations do not have the same number of cycles of each length they 
are not conjugate. Thus $S_{\pi}$ and $S_{\pi^{\prime}}$ are not isomorphic. However, for every $n>1$ and $n<-1, \pi^{n}$ is conjugate to $\left(\pi^{\prime}\right)^{n}$. Therefore, $\left(S_{\pi}\right)^{n}$ and $\left(S_{\pi^{\prime}}\right)^{n}$ are isomorphic.

Example 6.3. There exists a $K$ transformation with no roots of any order.

An example like this was first constructed by Clark [1]. Take $\pi$ to be a permutation of $\mathbf{Z}$ with no roots. If $R$ is a root of $S_{\pi}$ it is an automorphism of $S_{\pi}$. Then it would generate a permutation $\sigma$. The fact that $R^{n}=S_{\pi}$ implies $\sigma^{n}=\pi$.

Example 6.4. There exist two $K$ transformations which are weakly isomorphic, but not isomorphic. That is two transformations which are not isomorphic, but each one is a factor in the other.

We will give two different examples of this. The first is the simplest. The second one is more complicated but can be used to get to the transformations to be of finite entropy.

A) Take one transformation to be $S_{\pi}$ with $V=\mathbf{Z}$ and $\pi=i d$ and the other to be $S_{\pi} \bmod (1,2)$. They are not isomorphic because one has two coordinates associated but the other does not. One factor map is clear and the other takes a point of the form $\left(y_{1}, y_{2}, y_{3}, \ldots\right) \bmod (1,2)$ to $\left(y_{3}, y_{4}, y_{5}, \ldots\right)$

B) Take $V=\mathbf{Z} \times \mathbf{Z}$ and $\pi=i d$. $P$ will be generated by one permutation $p_{i}$ on the coordinates $(i, *)$ for every $i>0$. Call the $j$ th prime $a_{j}$. The permutation $p_{1}$ will be $(1,2)(3,4)(5,6)(7,8) \ldots$ and $p_{2}=(1,3,5,2,4,6)(7,8,9,10,11,12) \ldots$ Thus $p_{2}^{a_{2}}=p_{1}$. Inductively define $p_{n}^{a_{n}}=p_{n-1} . P^{\prime}$ will be the group generated by $P$ and $p_{1}$ acting on $(0, *)$.

Since $P^{\prime}$ is an extension of $P$, the factor generated by $P^{\prime}$ is clearly a factor of the one generated by $P$. The factor generated by $P$ is a factor of the factor generated by $P^{\prime}$ by the following map. Given a point $\left(b, x_{i, j}, \ldots\right) \bmod P$ map it to $\left(b, x_{i-1, j}, \ldots\right) \bmod P^{\prime}$. The two factors are not isomorphic because $P$ is generated by one permutation with order 2 while $P^{\prime}$ is generated by 2 permutations of order 2 .

Example 6.5. There exists a $K$ transformation which has an uncountable collection of two point factors which are not isomorphic.

Take $V=\mathbf{Z}$ and $\pi$ to be a permutation which has two cycles of every prime length. Take a sequence, $s_{i}$, of 0 's and 1 's indexed by the primes. For each sequence we get a permutation $p$ which interchanges the two cycles of length the $i$ th prime if $s_{i}=1$ and is the identity on those two cycles if $s_{i}=0$. Since each $p$ has order 2 , they all generate 2 point factors. If we take two different sequences of 0 's and 1's, then they differ for some $i$. Thus $\pi$ restricted to the unassociated coordinates of one factor has 2 cycles of the length of the $i$ th prime while the other factor does not. Thus any two different sequences generate factors which are not isomorphic.

Example 6.6. There exists a $K$ transformation with a countable collection of two point factors that are isomorphic, but do not sit the same.

We will construct this example by constructing a group of permutations which has countably many extensions of order 2 which are conjugate but no conjugation leaves the original group fixed. Let $\pi=i d$ on $V$ where

$$
V=\left(\mathbf{Z} \times\left(\bigcup_{1}^{\infty}[a(1, n), \ldots, a(2 n, n)]\right)\right) \cup\left[b_{1}, b_{2}, \ldots\right] .
$$


Define $p(i, n)$ to be

$((i, a(1,1)),(i, a(i, n+1))),((i, a(1,2)),(i, a(i, n+2))), \ldots,((i, a(1, n)),(i, a(i, 2 n)))$ and $p_{t}$ to be $\left(b_{1}, b_{t+1}\right),\left(b_{2}, b_{t+2}\right), \ldots,\left(b_{t}, b_{2 t}\right)$. For every integer $k$ there are infinitely many $p(i, n)$ that are $k$ linked two cycles. Let our group $P$ be the group generated by all the $p(i, n)$. The extension $P_{t}$ is generated by $P$ and $p_{t}$. All the $P_{t}$ are conjugate because for every integer $k$ there are infinitely many generators that are $k$ linked two cycles, thus they are all conjugate to $P$. No conjugation between $P_{i}$ and $P_{j}$ can preserve $P$ because $p_{i}$ and $p_{j}$ move a different number of the $b_{k}$. Now for any $t, S_{i d} \bmod P_{t}$ is a 2 point factor in $S_{i d} \bmod P . S_{i d} \bmod P_{i}$ and $S_{i d} \bmod$ $P_{j}$ are isomorphic since $P_{i}$ and $P_{j}$ are conjugate. They do not sit the same in $S_{i d}$ $\bmod P$ because no conjugation preserves $P$.

\section{Finite ENTROPy EXAMPLES}

In this section we show that we can construct all of our counterexamples with finite entropy $K$ transformations. In order to do this we need to show that our $K$ transformations satisfy the weak Pinsker property (WPP).

Lemma 7.1. The transformation $K$ satisfies the weak Pinsker property.

Proof. Take the factor $F_{n}$ which associates two points $x$ and $y$ if they have the same $n$ block structure. That is for all $i, K^{i}(x)$ is in an $n$ block if and only if $K^{i}(y)$ is in an $n$ block. For $n$ large enough, $F_{n}$ has arbitrarily small entropy. We will show that $K$ is relatively very weak Bernoulli with respect to each $F_{n}$. By Thouvenot's relative isomorphism theorem this shows that $K$ satisfies the weak Pinsker property.

The matching to show that $K$ is very weak Bernoulli with respect to $F_{n}$ is simple. Take any point in $F_{n}$ and any two pasts given $F_{n}$. The pasts may tell us what the first $n$ block after time 0 is, but they tell us nothing about any subsequent $n$ blocks. Thus we are free to match the futures conditioned on pasts $P_{1}$ and $P_{2}$ in exactly the same way.

In exactly the same way we can show that the base factor in all of the transformations $S_{\pi}$ satisfy the weak Pinsker property. In order to get examples of section 7 to be in transformations of finite entropy we must use the weak Pinsker property. We will now show that whatever properties our families of $K$ transformations had, there exist families of factors of finite entropy with the same properties.

Theorem 7.1. All of our counterexamples can be constructed with finite entropy transformations.

Proof. If we had $S_{\pi}$ and $S_{\pi^{\prime}}$ that are not isomorphic, then the factors we just described are not isomorphic. If they were, we could extend this map with an isomorphism of the Bernoulli compliments. Thus the only thing we need to show to get all of our examples in finite entropy transformations is that the appropriate weak isomorphisms, isomorphisms under time $n$, etc., still exist.

Group the set of coordinates $V$ into equivalence classes so that any two coordinates in the same cycle in $\pi$ or that are associated under $P$ are in the same equivalence class. Choose a positive function $e(v)$ that is constant on equivalence classes and $\sum_{V} e(v)<\infty$. For each equivalence class choose an $n$ such that the factor, $F_{n}$, which associates any two points that have the same $n$ block structure 
has entropy less that $e(v)$. Choose $f(v)$ to be such an $n$. Define $g(v)=g^{\prime}(\alpha(v))=$ $\max f(v), f(\alpha(v))$. The functions $g$ and $g^{\prime}$ define factors $G$ and $G^{\prime}$ which split off with Bernoulli compliments. This is done by associating points $\left(y_{1}, y_{2}, \ldots\right)$ and $\left(z_{1}, z_{2}, \ldots\right)$ if for all $v$ and all $n>g(v)$, the $n$ block structure of $y_{v}$ and $z_{v}$ are the same.

We have two transformations which are isomorphic (under time $n$ ). This is by the map $\Phi_{\alpha}$,

$$
\Phi_{\alpha}\left(y_{1}, y_{2}, \ldots\right) \bmod P=\left(y_{\alpha(1)}, y_{\alpha(2)}, \ldots\right) \bmod P^{\prime} .
$$

The paragraph above constructed factors $G$ and $G^{\prime}$ which have finite entropy. By construction $\Phi_{\alpha}$ takes $G$ to $G^{\prime}$. In this manner all the examples can be constructed with finite entropy examples.

\section{REFERENCES}

[1] Clark, Jack, A Kolmolgorov Shift with no Roots. Ph.D. Dissertation, Stanford University (1972).

[2] Feldman, Jacob, New $K$-automorphisms and a problem of Kakutani. Israel J. Math. 24 (1976), no. 1, 16-38. MR 53:13515

[3] Gerber, Marlies, A zero-entropy mixing transformation whose product with itself is loosely Bernoulli. Israel J. Math. 38 (1981), 1-22. MR 82d:28013

[4] Hoffman, Christopher, A loosely Bernoulli counterexample machine. to appear in Israel J. Math. (1997).

[5] Hoffman, Christopher, The behavior of Bernoulli shifts relative to their factors. to appear in $\operatorname{ETDS}(1997)$.

[6] Ornstein, Donald, Bernoulli shifts with the same entropy are isomorphic. Advances in Math. 4 (1970), 337-352. MR 41:1973

[7] Ornstein, Donald, Two Bernoulli shifts with infinite entropy are isomorphic. Advances in Math. 5 (1970), 339-348. MR 43:478a

[8] Ornstein, Donald, On the Root Problem in Ergodic Theory. Proc. Sixth Berkeley Symp. Math. Stat. Prob., Vol. II, University of California Press, 1967, pp. 347-356. MR 53:3259

[9] Ornstein, Donald, Ergodic Theory, Randomness and Dynamical Systems, Yale University Press, New Haven, (1974). MR 56:5836

[10] Ornstein, Donald; Shields, Paul, An uncountable family of $K$-automorphisms. Advances in Math. 10, (1973), 63-88. MR 52:3480

[11] Rudolph, Daniel, Two nonisomorphic $K$-automorphisms all of whose powers beyond one are isomorphic. Israel J. Math. 27 (1977), no. 3-4, 277-298. MR 56:3253

[12] Rudolph, Daniel, Two nonisomorphic $K$-automorphisms with isomorphic squares. Israel J. Math. 23 (1976), no. 3-4, 274-287. MR 54:2918

[13] Rudolph, Daniel, An example of a measure preserving map with minimal self-joinings, and applications. J. Analyse Math. 35 (1979), 97-122. MR 81e:28011

[14] Thouvenot, Jean-Paul, Quelques propriétés des systèmes dynamiques qui se décomposent en un produit de deux systèmes dont l'un est un schéma de Bernoulli. Israel J. Math. 21 (1975), no. 2-3, 177-207. MR 53:3263

The Hebrew University, Institute of Mathematics, Jerusalem, Israel

Current address: Department of Mathematics, University of Maryland, College Park, Maryland 20742

E-mail address: hoffman@math.umd.edu 